\title{
Laser Acceleration of Thin Flyers
}

\author{
Archie V. Farnsworth, Jr. \\ Sandia National Laboratories
}

\begin{abstract}
Laser energy delivered through an optical fiber has been used to accelerate a thin metallic foil to high velocity. Subsequent impact of the foil onto an explosive charge can initiate an explosion. The present computational study addresses the physical processes of laser absorption, energy transport, flyer acceleration, and foil impact on HE or on diagnostic materials in associated experiments. The objective has been to gain understanding that will allow optimizing the system for practical HE initiation. The structure of the foil, especially the presence of a thermally insulating layer near the ablation surface, significantly influences foil effectiveness as an initiator. These simulations show a marked effect on the acceleration process by that layer, which influences both the onset of full laser power absorption, and the physical competence of the foil on arrival at the HE. The effect on laser absorption is especially noteworthy at low laser intensity, where foil launch is marginal. A role of the glass fiber in absorbing laser energy and contributing ablated material is seen in the calculations and confirmed by experiments.
\end{abstract}

\section{Introduction}

This paper addresses the processes involved when laser energy is delivered through a small diameter optical fiber to a thin metallic foil in contact with the fiber end face, causing ablation and acceleration of that foil across a small gap to an explosive charge, for the purpose of initiating an explosion ${ }^{1,2}$. In this computational study, we have sought to understand these processes in sufficierit detail to allow a practical initiator of explosives (HE) to be designed and optimized. Another goal has been to develop the tools to provide some predictive capability for present and future studies of this subject.

\section{The Computational Tools}

In a previous paper ${ }^{1}$ we presented some numerical computations using the LASNEX hydrocode. Despite much excellent laser modeling capability in this code, it lacked capabilities important to this work: the capability to transmit laser energy through a transparent material, and to absorb and reflect the appropriate quantities of laser energy at a cold metal interface with such a material. We briefly describe here the CTH hydrocode ${ }^{3}$ and special additions to it that have been made to create a straight-forward laser energy transport and absorption package having the features required for this work.

The CTH hydrocode is an Eulerian code with a fixed grid which is distorted during a Lagrangian step, and then remapped back to the original grid for each hydrodynamic cycle. It contains numerous models ${ }^{3}$ for handling material properties and strength, and it accesses a substantial material property database. It also allows energy transport by thermal diffusion.

The additions to the code include a grid-following laser transport package that allows materials to be either transparent or absorbing. The absorption routines are appropriate to metallic absorption in cool materials, or plasma absorption when the materials become sufficiently hot. As laser energy is transported along a row of cells, each cell and material is treated as transparent, absorbing, or reflecting. If absorbing, energy is deposited in the cell and the balance conveyed to the next cell. If the material has a free electron number density that exceeds the critical value, energy is deposited in the vicinity as appropriate to a reflecting beam, and the direction of 


\section{DISCLAIMER}

This report was prepared as an account of work sponsored by an agency of the United States Government. Neither the United States Government nor any agency thereof, nor any of their employees, make any warranty, express or implied, or assumes any legal liability or responsibility for the accuracy, completeness, or usefulness of any information, apparatus, product, or process disclosed, or represents that its use would not infringe privately owned rights. Reference herein to any specific commercial product, process, or service by trade name, trademark, manufacturer, or otherwise does not necessarily constitute or imply its endorsement, recommendation, or favoring by the United States Government or any agency thereof. The views and opinions of authors expressed herein do not necessarily state or reflect those of the United States Government or any agency thereof. 


\section{DISCLAIMER}

Portions of this document may be illegible in electronic image products. Images are produced from the best available original document. 
transport is reversed for the reflected component. The unsolved physics problem of the ionization of dense vapors is avoided by using a simple criterion for transition between metallic and plasma-like behavior. We found sufficient accuracy for present purposes by using the plasma formulation when the densitytemperature product $\left(\rho_{0} / \rho\right) \sqrt{T}$ exceeded the value 2 , where $\rho_{0}$ and $\rho$ are the solid and current densities, and $\mathrm{T}$ is temperature measured in electron volts (eV). The ionization properties of metals are treated using routines from Lee and More ${ }^{4}$ and for non-metal plasmas by the simple perfect gas formula $z=(P A) /(\rho k T)-1$ where $\mathrm{z}$ is the ionization per atom, $P$ is pressure, $A$ is the average atomic mass, $\rho$ is the density, $k$ is the Boltzmann constant and $T$ is the temperature. The success of these simple relations derives in part from the fact that for the present problem the transition to the plasma state occurs very rapidly, once it begins.

\section{Physics Insights}

\section{The role of the glass fiber material}

When the code modifications described above became available, one of the noteworthy results that was seen immediately was the substantial role played by the glass substrate in the ablation process driving the metallic foil. Very soon after the formation of a plasma in the aluminum, the heat transported from the metal plasma to the glass produced a small amount of glass plasma. The laser light then began to deposit energy in glass, with only the residue reaching the foil. The process evolved with additional glass being heated so that the beginning point of absorption of laser power receded into the glass fiber during the laser pulse. Indeed, it was seen that for the typical laser conditions most of the absorption occurred in glass, and correspondingly, the glass provided most of the driving plasma. This unanticipated result has been confirmed by comparing the glass removed from the fiber after a shot with that predicted by the code to have been vaporized, with excellent agreement. Typical glass removal is about $0.4 \mu \mathrm{m}$. Additional confirmation is found in the spectroscopic evidence obtained by Trott ${ }^{5}$. The participation of the glass as a pusher of the foil suggested that some improvement in performance might be obtained by choosing a different substrate material for these experiments. Calculations were made using fused silica (the usual material), polycrystalline quartz, and a plastic (see Fig. 1). The performance of the foil using the

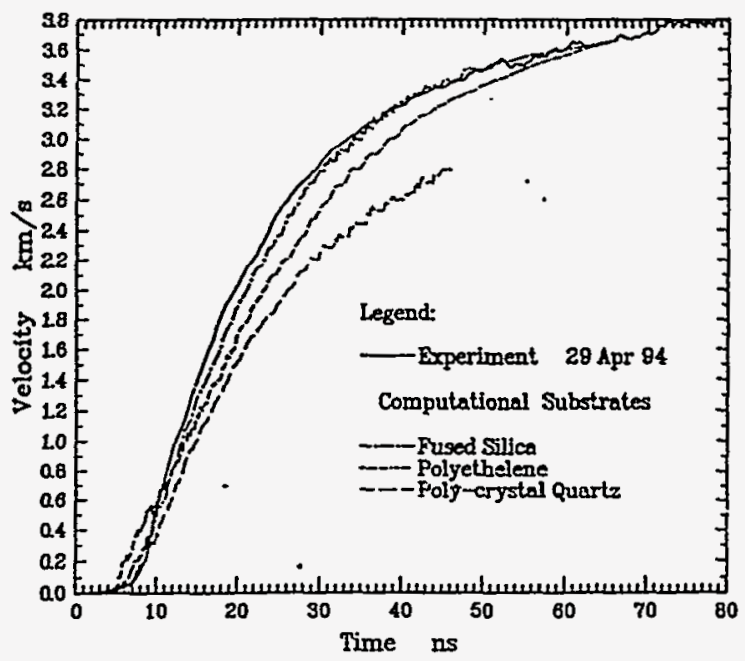

FIGURE 1. The velocity time history of a foil placed on a fused silica substrate (used in experiments) is compared with calculated results made assuming substrates of fused silica, polyethylene, and polycrystalline quartz.

polycrystalline quartz was much inferior to that obtained with the usual fused silica. The velocity obtained using polyethylene began early but lagged at intermediate times. It appears from the figure that it might exceed the velocity using fused silica at late times. For reasons that will be discussed below, it was thought undesirable to allow longer flight distances and longer times for these foils, so no present benefit was seen for changing the substrate material.

\section{The composite foil}

It has been observed in the experimental program that composite foils (consisting of a thin layer of aluminum followed by a thin layer of $\mathrm{Al}_{2} \mathrm{O}_{3}$, and the remainder of aluminum, with the first aluminum layer adjacent to the glass fiber) performed substantially better as impactors for igniting HE than do simple pure aluminum foils. Indeed, for very thin foils, experiments by $\mathrm{Harris}^{6}$ show failure to ignite the test $\mathrm{HE}$, even at quite large laser energies, while the composite foils consistently ignite the HE at moderate 
laser energy in comparable tests. Furthermore, at low incident laser energy, Trott ${ }^{2}$ has shown that the composite flyer is accelerated to much greater velocities than that achieved by the pure aluminum foil but that the velocity difference becomes negligibly small at larger energies. We believe that these observed phenomena can both be explained by results obtained from our computational simulations.

We first address the difference seen at larger incident laser energy. In Fig. 2, we show the temperature distributions in the aluminum impactor computed for each foil, at a time just before impact on HE would normally occur. The heating of the foil, of

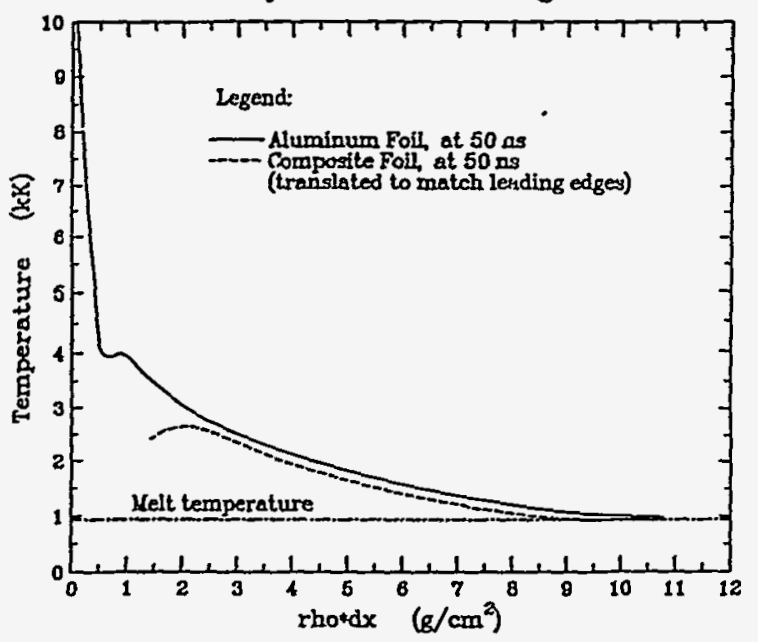

FIGURE 2. Temperature distributions of impactors for aluminum and composite foils near the time for impact on $\mathrm{HE}$.

course, continues throughout the entire flight time which is typically more than twice the laser pulse length (here the pulse width (fwhm) is $15 \mathrm{~ns}$ ).

The temperature of the pure aluminum foil is seen to be above the melt temperature throughout, while part of the composite foil remains at or below the melt temperature, at the impact side of the foil. A low density plasma accelerating a moiten metal at normal densities is clearly Rayleigh-Taylor unstable. One must expect the interpenetration of the materials at the gas-liquid interface. The presence of solid material in the composite case would clearly dampen the instability growth, resulting in a more competent impactor striking the HE. This interpretation is consistent with the findings of Harris, and also with impact velocity data of Trott ${ }^{2}$, who found a significant difference in the pulse width of the impact velocity records for the impacts of composite and aluminum foils onto a $\mathrm{LiF}$ window. The insulation afforded the impact layer by the thin layer of alumina between the metal layers delays the heating of the impactor, for the composite foil design.

The near melt conditions of the impactor computed for virtually all foils suggested trying a foil design that would minimize that condition. A short analytical survey of potential replacements for the impactor metal, emphasizing high melt temperatures was performed. Titanium was found to deliver results comparable to aluminum, from that study, and was tried as an impactor. The computations produced comparable results as an effective impactor, assuming a composite design in which the impactor aluminum was replaced with an equal mass of titanium. Using the ignition criterion of Setchell ${ }^{7}$ for fine grained HNS explosive, we produced the results shown in Figs. 3 and 4. Clearly, these results are comparable to

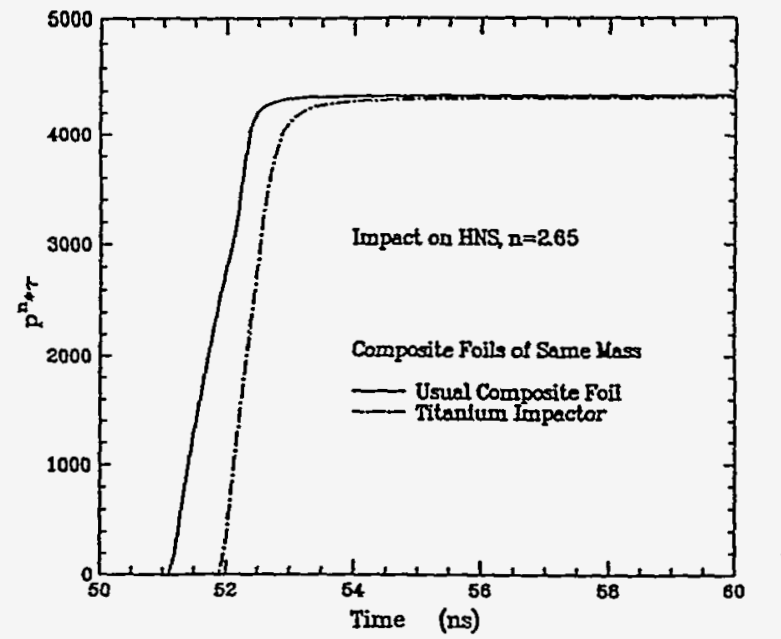

FIGURE 3. The integral of pressure in the explosive to the $n$th power times the time, as a function of time.

aluminum for impactor effectiveness, but the temperature of the titanium was not more favorable with respect to its melt temperature than was the aluminum; indeed, it is poorer in the computation, as seen in Fig. 4 despite the higher melt temperature.

We have noted previously ${ }^{1}$ that the onset of high level absorption begins with the formation of plasma at the metal-glass interface. Before that time, most of 
the energy is reflected back into the glass fiber. This fact is critical in understanding the higher velocity obtained by the composite foils in comparison with aluminum foils, for lower energy pulses.

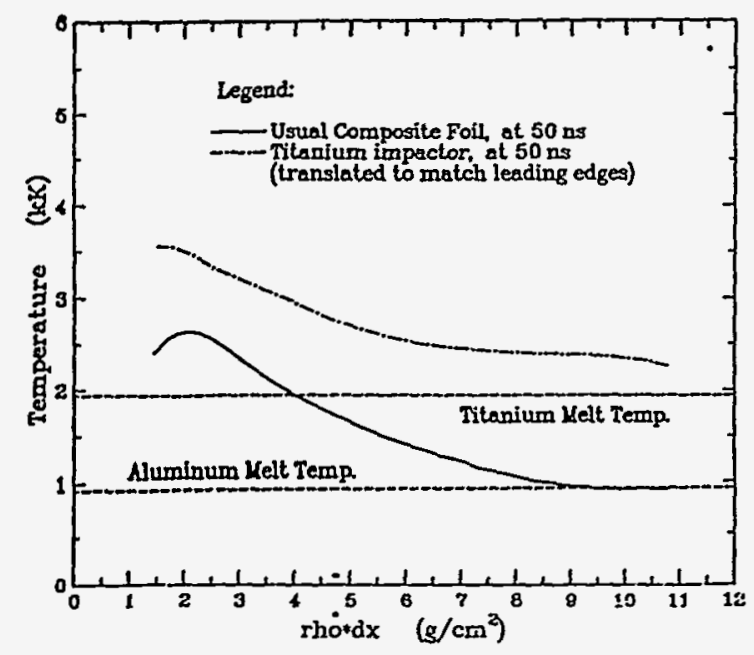

FIGURE 4. Temperatures of the impactors of composite foils having aluminum or titanium impactors, shown in relation to the melt temperatures for each material.

The timing of the onset of plasma creation is determined by the energy balance in the first metal layer; heating occurs by the small fraction of the input power that is not reflected back into the optical system $(\sim 4 \%$ here) and cooling by thermal conduction into the bulk of the foil. That energy balance was seen in the calculations to be affected by the thermally insulating layer of alumina at early time, and resulted

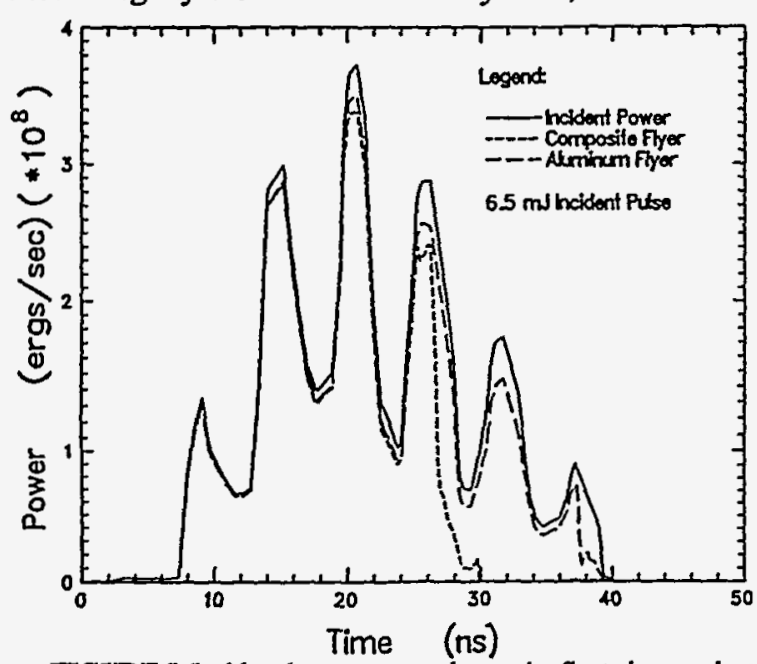

FIGURE 5. Incident laser power pulse, and reflected power lost (wasted) for the cases of the aluminum foil and the composite foil. in a highly absorbing plasma being formed at an earlier time during the input power pulse. This reduced the energy wasted from the first part of the pulse, and, for low energy pulses, resulted in a greatly enhanced velocity. In Fig. 5 the incident laser power and the computed reflected power are shown for both the aluminum and composite foils. The resultant peak flyer velocities are $45 \mathrm{~m} / \mathrm{s}$ and $600 \mathrm{~m} / \mathrm{s}$ respectively, a somewhat extreme example showing very marginal launch of the pure aluminum foil. This trend is consistent with Trott's velocity ${ }^{2}$ data, and with his measurements of reflected light for such cases.

\section{Conclusions}

These computations and their agreement with data have afforded insights that build confidence in our understanding of the processes underlying laser acceleration of thin foils. The CTH hydrocode, including present modifications for laser deposition, offer a tool with some actual predictive capability.

\section{Acknowledgments}

This work was performed at Sandia National Laboratories, supported by the U.S. Department of Energy, under contract DE-AC04-94AL85000. Gratitude is expressed for permission to quote the experimental findings of Steve Harris and Wayne Trott prior to their publication.

\section{References}

1. Farnsworth, A. V., Jr., and Lawrence, R. J.,"Numerical and Analytical Analysis of Thin Laser-Driven Flyers," in Shock Compression of Condensed Matter, 1991, pp. 821-824.

2. Trott, W. M. "Investigation of the Dynamic Behavior of LaserDriven Flyers," in Shock Compression of Condensed Matter, 1993, pp.1655-1658.

3. Hertel, E. S., Jr., Bell, R. L., Elrick, M. G., Fansworth, A. V., Kerley, G. I., McGlaun, J. M., Petne, S. V., Taylor, P. A., and Yarrington, P., "CTH: A Software Family for MultiDimensional Shock Physics Analysis", in Shock Waves @Marseille I, 1993, pp. 377-382.

4. Lee, Y. T., and More, R. M., Phys. Fluids 27, 1273-1286 (1984).

5. Trott, W. M. "High-Speed Optical Studies of the Driving Plasma in Laser Acceleration of Flyer Plate Studies".this vol.

6. Harris, S. M., Sandia National Lab., private communication.

7. Setchell, R. E. Sandia National Lab., private"communication. 